\title{
Comparison of Quality of Life Between Jordanian and Saudi Patients With Heart Failure
}

\author{
Mohannad Eid AbuRuz ${ }^{1} \&$ Fawwaz Alaloul $^{2}$ \\ ${ }^{1}$ College of Nursing, Applied Science Private University, Amman, Jordan \\ ${ }^{2}$ School of Nursing, University of Louisville, Louisville, KY, USA \\ Correspondence: Mohannad Eid AbuRuz, Associate Professor, Po Box 142 Shafa Badran, Amman11934, Jordan. \\ Tel: 96-279-026-2408; Fax: 96-265-232-899. E-mail: mohannadeid@yahoo.com; m_aburz@asu.edu.jo
}

Received: April 29, 2018 Accepted: June 3, 2018 Online Published: June 11, 2018

doi:10.5539/gjhs.v10n7p96 URL: https://doi.org/10.5539/gjhs.v10n7p96

\begin{abstract}
Introduction: Heart failure can influence all aspects of patients' health despite the improvement in its treatment. Different factors might affect the quality of life for patients with heart failure. These factors include but are not limited to: age, gender, ejection fraction, culture, and social support. Therefore, the purpose of this study was to examine differences in quality of life and perceived social support in patients with heart failure from Jordan and Saudi Arabia.
\end{abstract}

Materials and Methods: A cross-sectional correlational design was used to test the objective of this study. A total of 202 patients were recruited from outpatient clinics of three hospitals in Jordan and Saudi Arabia. Data were collected through the SF-36 and the MOS-SSS questionnaires.

Results: The results of this study demonstrated that Jordanian patients with heart failure reported significantly greater social support in all MOS-SSS subscales than the Saudi patients except for the tangible support. The Saudi patients reported significantly more mental impairment $(p<.01)$ and lower level of fatigue $(p<.01)$ than the Jordanian patients.

Conclusions: It is important to assess and identify physical and psychological resources available for patients at home and in the community to improve their quality of life.

Keywords: culture, heart failure, quality of life, social support, Jordan, Saudi Arabia

\section{Introduction}

Cardiovascular Disease (CVD) is the first leading cause of death worldwide (Mozaffarian et al., 2016). The prevalence of heart failure (HF) is 23 million worldwide and increasing, indicating that HF is a major global issue (Liu \& Eisen, 2014). The prevalence of HF in US is 5.70 million, and the incidence is 915,000 annually (Mozaffarian et al., 2016). It is estimated that the prevalence will increase by $46 \%$, and more than eight million Americans will have HF by the year 2030 (Mozaffarian et al., 2016). This will cost the government a burden of $\$ 69.70$ billion with an increase of $127.0 \%$ from 2012 (Mozaffarian et al., 2016). Heart failure was given as the major cause of death in $11.10 \%$ of US death certificates in 2015 (Mozaffarian et al., 2016). In 2013, HF was the underlying cause of death in 65,120 cases (Mozaffarian et al., 2016).

Other developed countries have nearly the same situation. For instance, there are 6.50 million HF patients in Europe and 2.50 million in Japan (Fotos et al., 2013). The incidence of HF in the United Kingdom is positively associated with the aging population; the incidence increased by $12.0 \%$ for those of 75 years of age and older (Riley, 2014). In Italy, HF accounts for $2.6 \%$ of all hospital admissions, and $4.0 \%$ of total length of hospital stay. Specifically, for CVD, HF was the primary case for admissions and length of stay with $27.5 \%$ and $40.5 \%$, respectively (Di Lenarda, 2002). In Africa, 44\% of the CVD incidence is due to HF which is higher than that of US and Europe (Mozaffarian et al., 2016). The prevalence of HF in Asia ranges from $1.26 \%$ to $6.70 \%$ (Mozaffarian et al., 2016).

Similar to the developed countries, HF is a critical health problem in developing countries (Pelegrino, Dantas, \& Clark, 2011). In Jordan, a developing Arabic country with an estimated population of 5.61 million, the prevalence of HF is approximately 100,000 cases; the estimated incidence is 8,251 cases annually (Health Grades Inc, 2012). 
Similarly, in Saudi Arabia, another developing Arabic country with an estimated population of 26.0 million, the prevalence of HF is approximately 456,000 cases and the estimated incidence is 32,200 cases annually (Inc., 2012). Heart failure interferes with all domains of patients' health despite the advancement in its treatment. It affects cardiovascular, neurological, and endocrine functions resulting in harmful outcomes. These negative outcomes are frequently due dyspnea, sleep disturbance, loss of energy, drowsiness and nervousness that are linked with HF (Heo, Doering, Widener, \& Moser, 2008). These signs and symptoms affects patients' ability to perform activates of daily living, working, sexuality, social and professional relationships, and finally impairing their Quality of Life (QOL) (Demir \& Unsar, 2011). For this study QOL is defined as a multidimensional construct that addresses the physical, role physical, role emotional, vitality, mental health, and social, aspects of life as perceived by the individuals. Therefore, an important purpose of treatment of HF is to improve QOL including symptom management.

It is well-known that there are many factors affecting the QOL for patients with HF. These factors include but are not limited to: age, gender, culture, social support, and marital status (AbuRuz, Alaloul, Saifan, Masa'deh, \& Abusalem, 2015; Alaloul, AbuRuz, Moser, Hall, \& Al-Sadi, 2016), functional class, duration of HF, left ventricular ejection fraction (LVEF), other comorbidities and psychosocial status (Chung, Moser, Lennie, \& Frazier, 2012). It has been shown that social support improved patients' health outcomes. Social support decreased morbidity, improved healthy lifestyles, and worked as a neutralizing agent against unpleasant life events (Staniute, Brozaitiene, \& Bunevicius, 2013) therefore improving QOL (Wang, Lau, Chow, Thompson, \& He, 2014). Social support is described as the perceived available emotional/informational, affectionate, tangible and positive social interaction social support (Sherbourne \& Stewart, 1991) .

One of the traditional religious customs among Muslim and Arabic cultures is to provide social support to persons when they are sick or in need for assistance (AbuRuz et al., 2015). The trajectory for HF patients is characterized by deterioration of physical functioning resulting in acute medical crises that often lead to hospitalization, dependency, and impairment in QOL (Huang et al., 2010). This situation requires continuous social support from the family which is a tradition in Jordan and Saudi Arabia as a result of their culture and religious customs. The religion of Islam encourages and directs Muslim caregivers to provide support for patients at all different stages of illness and recovery (Sarhill, LeGrand, Islambouli, Davis, \& Walsh, 2001; Wehbe-Alamah, 2008).

Jordan and Saudi Arabia are approximately homogenous cultures; however, cultural differences exist even within countries that were previously fairly homogenous (Huang et al., 2010). Although Saudi Arabia and Jordan are similar in terms of religion and culture, they differ in the cost of living, weather conditions, and the role of religion. The role of religion in Saudi Arabia is more prominent than in Jordan. In Saudi Arabia, religion is interwoven with all aspects of life including legal/governmental issues. The cost of living in Jordan is more expensive than in Saudi Arabia (NUMBEO, ND). For example, the average cost of a gallon of gasoline in Saudi Arabia is \$.24 compared to $\$ 1.20$ in Jordan ("Global Petrol Prices. Saudi Arabia gasoline prices, liter. ," 2018). Prior research has shown that physical inactivity in adult Saudis was higher than that for adult Jordanians (Awadalla NJ, 2014; Bawadi, Khader, Haroun, Al-Omari, \& Tayyem, 2011). The health care system in Saudi Arabia mainly relies on foreign healthcare providers including providers from Western countries who speak different languages and have different culture (Albejaidi, 2010). Therefore, it is important to acknowledge differences in patients from different countries who share the same religion and culture.

Studies that compare the QOL between/among countries that are different in terms of religion or culture are lacking. Only one study was found which compared the QOL between American and Taiwanese HF patients (Huang et al., 2010). To our knowledge, there have been no studies on the differences of QOL of HF patients among Arabic developing countries. Therefore, the purpose of this study was to examine differences and similarities in QOL and perceived social support in patients with HF from Jordan and Saudi Arabia using the Short Form-36 version 2 (SF-36); and the Medical Outcomes Study-Social Support Survey (MOS-SSS) (Sherbourne \& Stewart, 1991).

The specific aims of the study were to: (a) compare scores of Jordanian and Saudi patients with HF on the eight domains of QOL, Physical Component Summary (PCS), and Mental Component Summary (MCS) of the SF-36; (b) compare Jordanian and Saudi patients with HF on the four MOS-SSS subscales; and (c) identify the best predictive models for the PCS and the MCS in patients with HF from Jordan and Saudi Arabia separately.

\section{Methods}

\subsection{Design, Sample, and Setting}

A cross-sectional correlational design was used in the study. A total of 202 patients participated--99 from Jordan 
and 103 from Saudi Arabia. The patients were recruited from outpatient clinics of a nonprofit hospital and a teaching hospital in Jordan and one tertiary care hospital in the Eastern province in Saudi Arabia. These are referral hospitals which treat patients with HF from all areas of the two countries. Patients who met the following inclusion criteria and agreed to participate in the study were included: a confirmed diagnosis of HF and able to read and write Arabic. Patients were excluded from the study if they had another chronic disease that affected their QOL (i.e., cancer, liver failure, and renal failure, chronic obstructive pulmonary disease). Patients were not excluded from the study based on the stage of heart failure. Sample size was calculated based on $\mathrm{G}^{*}$ power software, using the following assumptions: a) multiple regression test with 9 predictors, b) alpha of .01, c) power of .9, and d) medium effect size of .15. Based on these assumptions, the needed sample size was 186 participants.

\subsection{Instruments}

\subsubsection{The Short Form-36 Version 2 (SF36)}

This 36-item instrument measures QOL and consists of eight scales/domains: physical functioning (10 items), role physical functioning (four items), role emotional functioning (three items), vitality (four items), mental health (five items), social functioning (two items), body pain (two items), general health (five items), and one item comparing current patient's health with the last year (Ware \& Sherbourne, 1992). The scores for these domains ranged from 0-100, higher scores indicate improved QOL.

Two major summaries can be calculated: Physical Component Summary (PCS), representing; physical functioning, role physical functioning, body pain, and general health, and Mental component summary (MCS), representing; role emotional functioning, vitality, mental health, and social functioning. PCS and MCS have values ranged from $0-100$. There are no published mean norms for this instrument in Arabic populations. Therefore, we used a cutoff point of 50 to define impairment in each domain based on the 1998 US general population cutoff (Alaloul et al., 2016). This instrument has been translated into Arabic and has shown acceptable psychometric properties (Coons, Alabdulmohsin, Draugalis, \& Hays, 1998). The Arabic version of this instrument also showed acceptable psychometric properties in a sample of Arab patient with HF. The minimum reliability coefficient for bodily pain was .71 and the highest was for social functioning (.95) (Alaloul et al., 2016). Cronbach's alphas for the eight scales in this study were: .88 ; for physical functioning .87 , for role physical .83 , for social functioning .75 , for role emotional .70, for bodily pain .79, for general health .77, vitality 071, and for mental health 69 .

\subsubsection{Medical Outcomes Study-Social Support Survey (MOS-SSS)}

This 19-item Likert scale questionnaire is designed to assess perceived functional support (Sherbourne \& Stewart, 1991). For each item, scores ranged from 1 (none of the time) to 5 (all of the time). The scores were standardized by transforming them into a 0-100 scale. The first 18 items are divided into four subscales: emotional/informational support (eight items), affectionate support (three items), tangible support (four items) and positive social interaction (three items). Item number 19 measures the availability of people to support respondents and keep their minds at peace. Higher scores indicate higher levels of support for the subscales and for the overall support index (Sherbourne \& Stewart, 1991). Sherbourne and Stewart (1991) used a large sample of 2,987 patients with chronic conditions to test psychometric proprieties of this instrument. The minimum internal consistency coefficient was positive social interaction $(\alpha=.91)$ and the highest coefficient was emotional/informational support scale $(\alpha=.96)$. The overall reliability coefficient was .97 . Moreover, the instrument showed a strong convergent and discriminate validity (Sherbourne \& Stewart, 1991) [23]. Cronbach's alphas ranged from .79 to .87 for the Arabic translation of this instrument in a sample of 63 Arab post bone marrow transplant (Alaloul, 2007).

\subsubsection{Demographic and Medical Characteristics}

A socio-demographic list was developed specifically to collect data on participants' age, gender, marital status, educational level, employment status and annual income. Clinical variables including time since diagnosis with HF, LVEF, history of diabetes, or hypertension were abstracted from patients' medical records. Left ventricular ejection fraction is an indicator of systolic function (Meta-analysis Global Group in Chronic Heart, 2012)

\subsection{Data Collection Procedure}

The study was approved by the Institutional Review Board committee at the Applied Science Private University, Amman, Jordan, and from the three hospitals where data were collected. After approval, the principal investigator met with the cardiologists and the nursing directors and gave a detailed description of the study and its objectives. Patients were identified by their cardiologists. Patients with heart failure who met the inclusion criteria were contacted by the research assistants during their clinic visit and signed an informed consent after a thorough explanation about the study. The Arabic versions of the SF-36, the MOS-SSS and the demographic data form were completed by the participants in a calm, confidential place in the hospital. Three research assistants obtained 
clinical variables data from patients' medical records. All paper copies of the questionnaires were kept in a locked cabinet in the investigators' office. The files on the computer were password protected as well. Data was collected between June and November, 2017.

\subsection{Data Analysis}

Statistical Package for the Social Sciences for Windows 21.0 (SPSS, Inc., Chicago, Illinois) was used to analyze the data. To be statistically significant, an alpha of less than .05 was set a priori. To characterize the sample, descriptive statistics including means, standard deviations, frequencies, and percentages were used. T-tests or chi-square tests were used to examine differences in individual characteristics between Jordanian and Saudi patients. To examine differences in QOL domains and the four social support subscales scores between Jordanian and Saudi patients with HF, t-tests were used. Separate multiple linear regressions were run to identify predictors of PCS and MCS in both Jordanian and Saudi samples of patients with HF. The following variables were entered into the regression models: age, gender, marital status, employment status, income, LVEF, length of HF diagnosis, diagnosis of diabetes, and diagnosis of hypertension.

\section{Results}

Sample characteristics are presented in Table 1. Jordanian patients were older, fewer were employed, more were married, and they had a lower income than the Saudi patients.

Table 1. Sociodemographic and Clinical Characteristics of the Sample

\begin{tabular}{|c|c|c|c|}
\hline \multirow{2}{*}{ Characteristics } & \multicolumn{3}{|c|}{ Mean \pm SD or Frequency (\%) } \\
\hline & Total $(\mathrm{N}=\mathbf{2 0 2})$ & Jordanian $(n=99)$ & Saudi $(n=103)$ \\
\hline Age (yrs) & $53.51 \pm 14.41$ & $56.9 \pm 11.31$ & $50.32 \pm 16.30^{*}$ \\
\hline Male & $124(61.38)$ & $64(64.64)$ & $60(58.325)$ \\
\hline \multicolumn{4}{|l|}{ Marital status } \\
\hline Married & $148(73.26)$ & $85(85.85)$ & $63(61.16)^{*}$ \\
\hline Unmarried & $54(26.74)$ & $14(14.15)$ & $40(38.84)^{*}$ \\
\hline \multicolumn{4}{|l|}{ Employment } \\
\hline Employed & $111(54.95)$ & $37(37.37)$ & $74(71.84)^{*}$ \\
\hline Not employed & $91(45.05)$ & $62(62.63)$ & $29(28.16)^{*}$ \\
\hline \multicolumn{4}{|l|}{ Income/month } \\
\hline$<\$ 1300$ & $127(62.87)$ & $80(80.80)$ & $47(45.63)^{*}$ \\
\hline$\geq 1301 \$$ & $75(37.1)$ & $19(19.2)$ & $56(54.4)^{*}$ \\
\hline Ejection Fraction & $38.21 \pm 5.70$ & $37.82 \pm 5.81$ & $38.62 \pm 5.61$ \\
\hline Length of HF Diagnosis & $4.50 \pm 3.01$ & $3.60 \pm 2.30$ & $5.40 \pm 3.30^{*}$ \\
\hline Diagnosis of diabetes & $84(41.60)$ & $35(35.35)$ & $49(47.57)$ \\
\hline Diagnosis of hypertension & $144(71.28)$ & $69(69.69)$ & $75(72.81)$ \\
\hline
\end{tabular}

The results comparing differences in the eight domains of QOL, PCS, and MCS between Jordanian and Saudi patients with HF are presented in Table 2. The scores of the Jordanian patients for all QOL domains and the PCS and MCS were lower than 50 except for mental health. The scores for Saudi patients' for all QOL domains were lower than 50 except for vitality. When the two groups were compared on QOL domains, Saudi patients had scored higher in role physical and vitality domains. However, Jordanian patients reported better general health, mental health, and MCS. There were no significant differences in any other domains. The results comparing differences in the four MOS-SSS subscales between Jordanian and Saudi patients with HF are presented in Table 3. Jordanian patients have higher support in all subscales, except for tangible support. 
Table 2. Comparison of Means of the Eight Domains of QOL, Physical Component Summary (PCS), and Mental Component Summary (MCS) between Jordanian and Saudi Patients with Heart Failure, (N=202)

\begin{tabular}{lll}
\hline Subscale/Domain & Jordanian (n=99) & Saudi (n=103) \\
\hline Physical Functioning (Level of limitation in physical activities) & $38.91 \pm 23.70$ & $42.91 \pm 14.10$ \\
Role Physical (Problems with usual role related to physical health) & $36.40 \pm 24.61$ & $43.21 \pm 11.52^{*}$ \\
Social Functioning (Interference with social activities) & $42.82 \pm 25.21$ & $45.01 \pm 10.71$ \\
Role Emotional (Problems with usual role related to emotional health) & $42.33 \pm 26.54$ & $45.24 \pm 9.63$ \\
Bodily Pain (Level of pain) & $37.95 \pm 21.94$ & $33.91 \pm 11.52$ \\
General Health (General perception about health) & $40.87 \pm 15.10$ & $26.70 \pm 12.51^{*}$ \\
Vitality (Energy and fatigue) & $34.81 \pm 19.70$ & $51.62 \pm 6.82^{*}$ \\
Mental Health (Level of psychological status) & $53.41 \pm 8.30$ & $45.05 \pm 10.70^{*}$ \\
Physical component summary (PCS) & $34.06 \pm 8.44$ & $36.71 \pm 10.60$ \\
Mental component summary (MCS) & $48.81 \pm 9.90$ & $33.81 \pm 6.50^{*}$
\end{tabular}

$* p<.01$.

Table 3. Comparison of Means of the Four Subscales of the Social Support between Jordanian and Saudi Patients with Heart Failure, $(\mathrm{N}=202)$.

\begin{tabular}{lll}
\hline Scale/Subscale & Jordanian $(\mathbf{n}=\mathbf{9 9})$ & Saudi $(\mathbf{n}=\mathbf{1 0 3})$ \\
\hline Emotional/Information Support & $65.01 \pm 19.81$ & $51.62 \pm 7.32^{*}$ \\
Tangible Support & $81.63 \pm 13.40$ & $87.54 \pm 9.33^{*}$ \\
Affectionate Support & $86.33 \pm 13.21$ & $73.30 \pm 9.93^{*}$ \\
Positive Social Interaction & $75.42 \pm 12.41$ & $63.84 \pm 10.33^{*}$ \\
Total score & $73.74 \pm 18.15$ & $63.64 \pm 7.92^{*}$ \\
\hline
\end{tabular}

$* p<.01$.

The results of the regression analyses that identified the best predictive models for PCS and MCS in patients with HF from Jordan and Saudi Arabia are presented in Tables 4 and 5, respectively. Ejection fraction was the only significant predictor in both samples. Tangible support was an independent predictor for PCS in both samples.

Table 4. Regression Analyses: Predictors of the SF-36 Physical Component Summary (PCS) and Mental Component Summary (MCS) in Jordanian Patients $(n=99)$

\begin{tabular}{llll}
\hline Outcomes/Predictors & Standardized $\boldsymbol{\beta}$ & $\boldsymbol{t}$ & Model Statistics \\
\hline Physical component summary & & & \\
Ejection Fraction & 0.36 & 4.44 & $R^{2}=0.45 ; F_{(4,94)}=19.0$, \\
Gender & 0.26 & 3.23 & $p<.001$ \\
History of Diabetes & -0.18 & -2.31 & \\
Tangible Support & 0.41 & 5.25 & \\
\hline Mental component summary & & & $R^{2}=0.10 ; F_{(2,96)}=4.75$, \\
$\quad$ Ejection Fraction & 0.21 & 3.12 & $p<.05$ \\
History of Diabetes & -0.17 & -1.84 & \\
\hline
\end{tabular}


Table 5. Regression Analyses: Predictors of the SF-36 Physical Component Summary (PCS) and Mental Component Summary (MCS); Saudi Patients $(n=103)$

\begin{tabular}{clll}
\hline Outcomes/Predictors & Standardized $\boldsymbol{\beta}$ & $\boldsymbol{t}$ & \multicolumn{1}{c}{ Model Statistics } \\
\hline Physical component summary & & & $R^{2}=0.45 ; F_{(2,100)}=40.5$, \\
Ejection Fraction & 0.53 & 6.81 & 001 \\
Tangible Support & 0.28 & 3.61 & \\
\hline Mental component summary & & $R^{2}=0.41 ; F_{(3,99)}=24.4$, \\
Ejection Fraction & 0.25 & 3.11 & $p<.001$ \\
Age & -0.35 & -4.23 & \\
Emotional support & 0.28 & 3.41 & \\
\hline
\end{tabular}

\section{Discussion}

There is a need for research to identify any significant areas of difference in QOL among Arab patients with HF from two Arab countries. Despite similarities between the two countries in terms of culture and religion, they are different in terms of economy, weather, and role of religion in life. The cost of living in Jordan is higher than Saudi Arabia (NUMBEO, ND). The climate in Saudi Arabia is hotter and dryer than Jordan. In addition, patients with HF are at higher risk for heat intolerance due to the impairment in sweating due to heat stress (Cui \& Sinoway, 2014). Although both countries share the same religion and culture, other factors may influence their health outcomes including QOL. Therefore, healthcare providers should recognize the importance of individualizing healthcare delivery.

The results of this study demonstrated a significance difference in perceived social support between Jordanian and Saudi patients. Jordanian patients with HF reported significantly greater social support than the Saudi patients except for the tangible support. This difference in tangible support may be related to the availability of domestic paid workers and drivers in Saudi Arabia (Schwenken \& Heimeshoff, 2011). In addition to the family members, domestic workers provide tangible support, including doctor visits, to the Saudi patients with HF. Domestic paid workers are responsible for cleaning, taking care of children and elderly, gardening, driving and other services provided in private households (Schwenken \& Heimeshoff, 2011). As a result of the low economic status, Jordanian patients mainly received tangible support from their family and consequently they had to do more household work. Saudi families may depend on housemaids and drivers to provide support to HF patients. Housemaids generally do not speak the Arabic language fluently and come from different cultures and consequently are unable to provide other types of support. In addition, more than one-quarter of the Saudi patients who participated in this study lived without spouses. Therefore, healthcare providers are required to evaluate the availability and effectiveness of social support resources in home and community using valid and reliable measures.

Patients with HF in both countries reported poor QOL in all domains, general physical and mental health status in comparison to the US general population norms of the SF-36v2 (Maglinte, Hays, \& Kaplan, 2012). The Saudi patients reported significantly more psychological impairment than the Jordanian patients. This difference in psychological status between Saudi and Jordanian patient with HF may be due to significant differences in perceived emotional, affectionate, and positive social interaction support. On the other hand, Jordanian patients reported higher level of fatigue. A possible explanation for this difference is that Saudi patients received a higher level of tangible support. Several studies conducted in Arab and Western countries showed inconsistent findings regarding the relationship between social support and QOL (Alaloul et al., 2016; Barutcu \& Mert, 2013).

This study also investigated demographic and clinical variables associated with QOL in both Jordanian and Saudi patients with HF. Ejection fraction was a predictor of all QOL domains among both Saudi and Jordanian patients with HF. Several studies showed that proper management of reduced left ventricular ejection fraction can enhance QOL (Vorilhon et al., 2016). Therefore, healthcare providers are encouraged to use different modalities to improve ejection fraction which may lead to improvement in QOL. Several demographic characteristics were associated with higher levels of QOL. Being female was a strong predictor in Jordanian patients while being older in Saudi patients was associated with low QOL. Therefore, healthcare provides should not only focus on clinical factors but also socio-demographic factors in addressing and improving patients' QOL. 


\section{Implications for Practice}

Healthcare providers should not assume that all patients from the same culture or religion have the same culture and religious needs. Healthcare providers should consider the socio-economic status of patients with HF. It is important to assess and identify physical and psychological resources available for patients at home and in the community. A better understanding of these gaps in resources available to the patients may help healthcare providers direct patients to available resources in the community. Helping patients identify and access available physical and psychological resources may help meet their needs and consequently improve their QOL, decrease mortality and morbidity. Designing a culturally specific assessment tools may help to identify QOL and social supports resources of these patients. Nurses are in a unique position to assist Jordanian and Saudi family members in providing social support for HF patients via conferences on their role in improving QOL. In addition to the important role of culture and religion, health care providers should also personalize delivery of care to individual patients. In this study, data on housemaids and drivers were not collected. Future research should include data on housemaid and drivers on the demographic form to have a better understanding of tangible support. Further research is needed to understand the relationship between social support and QOL among patients with HF from different countries and culture.

\section{Limitation of the Study}

The major limitations of this study was the cross-sectional design which limit our understanding of factors that predicting patients' QOL over time and the convenience sampling technique used in this study. Moreover, the staging of the HF was not considered in this study due to the lack of this information in the medical records which might have an effect on understanding the QOL for patients living with HF.

\section{Conclusion}

The Saudi patients reported significantly more psychological impairment and lower level of fatigue than the Jordanian patients. Results of this study also highlight the importance of understanding the effects of economic status on QOL and social support in patients with HF. Providing HF patients with the appropriate physical and psychological support may have a great impact on patients' QOL and other healthcare outcomes.

\section{Acknowledgements}

The authors are grateful to the Applied Science Private University and Hashemite University in Jordan, for the financial support granted to this research project.

\section{Competing Interests Statement}

The authors declare that there are no competing or potential conflicts of interest.

\section{References}

AbuRuz, M. E., Alaloul, F., Saifan, A., Masa'deh, R., \& Abusalem, S. (2015). Quality of Life for Saudi Patients With Heart Failure: A Cross-Sectional Correlational Study. Glob J Health Sci, 8(3), 49-58. https://doi.org/10.5539/gjhs.v8n3p49

Alaloul, F. (2007). Examination of the validity of the Arabic version of the Medical Outcome Study (MOS) Social Support Survey using confirmatory factor analysis. (Master Quantitative MS Thesis), University of Kentucky, Lexington.

Alaloul, F., AbuRuz, M. E., Moser, D. K., Hall, L. A., \& Al-Sadi, Ahmad. (2017). Factors associated with quality of life in Arab patients with heart failure. Scandinavian Journal of Caring Sciences, 31 (1), 104-111 https://doi.org/10.1111/scs.12324

Albejaidi. (2010). Healthcare system in Saudi Arabia: An analysis of structure, total quality management and future challenges. Journal of Alternative Perspectives in the Social Sciences, 2(2), 794-818.

Awadalla, N. J., Aboelyazed, A. E., Hassanein, M. A., Khalil, S. N., Aftab, R., Gaballa, II, \& Mahfouz, A. A. (2014). Assessment of physical inactivity and perceived barriers to physical activity among health college students, south-western Saudi Arabia. East Mediterranean Health Journal, 20(10), 596-604.

Barutcu, C. D., \& Mert, H. (2013). The relationship between social support and quality of life in patients with heart failure. J Pak Med Assoc, 63(4), 463-467.

Bawadi, H. A., Khader, Y. S., Haroun, T. F., Al-Omari, M., \& Tayyem, R. F. (2011). The association between periodontal disease, physical activity and healthy diet among adults in Jordan. $J$ Periodontal Res, 46(1), 74-81. https://doi.org/10.1111/j.1600-0765.2010.01314.x 
Chung, M. L., Moser, D. K., Lennie, T. A., \& Frazier, S. K. (2012). Perceived social support predicted quality of life in patients with heart failure, but the effect is mediated by depressive symptoms. Qual Life Res, 22(7), 1555-1563. https://doi.org/10.1007/s11136-012-0294-4

Coons, S. J., Alabdulmohsin, S. A., Draugalis, J. R., \& Hays, R. D. (1998). Reliability of an Arabic version of the RAND-36 Health Survey and its equivalence to the US-English version. Med Care, 36(3), 428-432.

Cui, J., \& Sinoway, L. I. (2014). Cardiovascular responses to heat stress in chronic heart failure. Curr Heart Fail Rep, 11(2), 139-145. https://doi.org/10.1007/s11897-014-0191-y

Demir, M., \& Unsar, S. (2011). Assessment of quality of life and activities of daily living in Turkish patients with heart failure. Int J Nurs Pract, 17(6), 607-614. https://doi.org/10.1111/j.1440-172X.2011.01980.x

Di Lenarda, Goliani P, Grella M, Sabbadini G, Driussi M, Sinagra G. (2002). Hospital statistics as tool in epidemiologic studies: heart failure in Trieste. Italian Heart Journal, 3(1), 58-70.

Fotos, N. V., Giakoumidakis, K., Kollia, Z., Galanis, P., Copanitsanou, P., Pananoudaki, E., \& Brokalaki, H. (2013). Health-related quality of life of patients with severe heart failure. A cross-sectional multicentre study. Scand $J$ Caring Sci, 27(3), 686-694. https://doi.org/10.1111/j.1471-6712.2012.01078.x

Global Petrol Prices. Saudi Arabia gasoline prices, liter. (2018). Retrieved from http://www.globalpetrolprices.com/Saudi-Arabia/gasoline_prices/.

Health Grades Inc. (2012). Statistics by country for congestive heart failure. Retrieved from http://cureresearch.com/c/congestive_heart_failure/stats-country.htm

Heo, S., Doering, L. V., Widener, J., \& Moser, D. K. (2008). Predictors and effect of physical symptom status on health-related quality of life in patients with heart failure. Am J Crit Care, 17(2), 124-132. https://doi.org/17/2/124

Huang, T. Y., Moser, D. K., Hwang, S. L., Lennie, T. A., Chung, M., \& Heo, S. (2010). Comparison of health-related quality of life between American and Taiwanese heart failure patients. $J$ Transcult Nurs, 21(3), 212-219. https://doi.org/1043659609358779

Inc., Health Grades. (2012). Statistics by Country for Congestive Heart Failure.

Liu, L., \& Eisen, H. J. (2014). Epidemiology of heart failure and scope of the problem. Cardiol Clin, 32(1), 1-8, vii. https://doi.org/S0733-8651(13)00092-1

Maglinte, G. A., Hays, R. D., \& Kaplan, R. M. (2012). US general population norms for telephone administration of the SF-36v2. J Clin Epidemiol, 65(5), 497-502. https://doi.org/S0895-4356(11)00309-X

Meta-analysis Global Group in Chronic Heart, Failure. (2012). The survival of patients with heart failure with preserved or reduced left ventricular ejection fraction: an individual patient data meta-analysis. European Heart Journal, 33(14), 1750-1757. https://doi.org/10.1093/eurheartj/ehr254

Mozaffarian, D., Benjamin, E. J., Go, A. S., Arnett, D. K., Blaha, M. J., Cushman, M., .. Turner, M. B. (2016). Heart Disease and Stroke Statistics-2016 Update: A Report From the American Heart Association. Circulation, 133(4), e38-360. https://doi.org/CIR.0000000000000350

NUMBEO. (ND). Cost of Living Comparison Between Saudi Arabia and Jordan. Retrieved from https://www.numbeo.com/cost-of-living/compare_countries_result.jsp?country $1=$ Saudi+Arabia\&country2= Jordan

Pelegrino, V. M., Dantas, R. A. S., \& Clark, A. M. (2011). Health-related Quality of Life Determinants in Outpatients With Heart Failure'. Rev. Latino-Am. Enfermagem, 19(3), 451- 457.

Riley, J. (2014). Cognitive dysfunction and its impact on the person with heart failure. British Journal of Cardiac Nursing, 9(8), 382-387.

Sarhill, N., LeGrand, S., Islambouli, R., Davis, M. P., \& Walsh, D. (2001). The terminally ill Muslim: death and dying from the Muslim perspective. American Journal of Hospice and Palliative Care, 18(4), 251-255. https://doi.org/10.1177/104990910101800409

Schwenken, H., \& Heimeshoff, L. M. (2011). Domestic Workers Count: Global Data on an Often Invisible Sector: Kassel University Press.

Sherbourne, C. D., \& Stewart, A. L. (1991). The MOS social support survey. Soc Sci Med, 32(6), 705-714.

Staniute, M., Brozaitiene, J., \& Bunevicius, R. (2013). Effects of social support and stressful life events on 
health-related quality of life in coronary artery disease patients. $J$ Cardiovasc Nurs, 28(1), 83-89. https://doi.org/10.1097/JCN.0b013e318233e69d

Vorilhon, C., Jean, F., Mulliez, A., Clerfond, G., Pereira, B., Sapin, V., . . Eschalier, R. (2016). Optimized management of heart failure patients aged 80 years or more improves outcomes versus usual care: The HF80 randomized trial. Arch Cardiovasc Dis, 109(12), 667-678. https://doi.org/S1875-2136(16)30170-X

Wang, W., Lau, Y., Chow, A., Thompson, D. R., \& He, H. G. (2014). Health-related quality of life and social support among Chinese patients with coronary heart disease in mainland China. Eur J Cardiovasc Nurs, 13(1), 48-54. https://doi.org/1474515113476995

Ware, J. E., Jr., \& Sherbourne, C. D. (1992). The MOS 36-item short-form health survey (SF-36). I. Conceptual framework and item selection. Med Care, 30(6), 473-483.

Wehbe-Alamah, H. (2008). Bridging generic and professional care practices for Muslim patients through use of Leininger's culture care modes. Contemporary Nurse, 28(1-2), 83-97.

\section{Copyrights}

Copyright for this article is retained by the author(s), with first publication rights granted to the journal.

This is an open-access article distributed under the terms and conditions of the Creative Commons Attribution license (http://creativecommons.org/licenses/by/4.0/). 